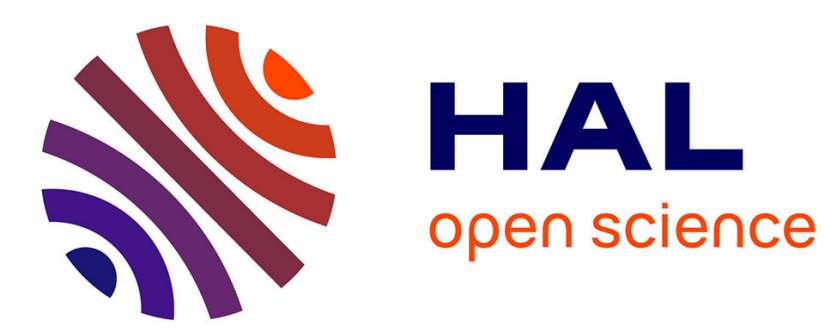

\title{
Single-Layer Reflectarray Cell Synthesis Based on Filter Synthesis Techniques
}

Alexandre Grossetete, Erwan Fourn, Raphaël Gillard

\section{To cite this version:}

Alexandre Grossetete, Erwan Fourn, Raphaël Gillard. Single-Layer Reflectarray Cell Synthesis Based on Filter Synthesis Techniques. 2016 Loughborough Antennas \& Propagation Conference (LAPC), Nov 2016, Loughborough, United Kingdom. 10.1109/LAPC.2016.7807481 . hal-01430635

\section{HAL Id: hal-01430635 https://hal.science/hal-01430635}

Submitted on 10 Jan 2017

HAL is a multi-disciplinary open access archive for the deposit and dissemination of scientific research documents, whether they are published or not. The documents may come from teaching and research institutions in France or abroad, or from public or private research centers.
L'archive ouverte pluridisciplinaire HAL, est destinée au dépôt et à la diffusion de documents scientifiques de niveau recherche, publiés ou non, émanant des établissements d'enseignement et de recherche français ou étrangers, des laboratoires publics ou privés. 


\title{
Single-Layer Reflectarray Cell Synthesis Based on Filter Synthesis Techniques
}

\author{
A.GROSSETETE, E.FOURN, R.GILLARD \\ Institute of Electronics and Telecommunications of Rennes \\ Rennes, France \\ Email: Alexandre.grossetete@insa-rennes.fr
}

\begin{abstract}
This paper presents a method for synthesizing the equivalent circuit of a single-layer reflectarray unit-cell based on filter synthesis techniques. Metallic-ring and slot-ring cells are synthesized respectively by $3^{r d}$-order band-stop and bandpass Tchebycheff filters. A phase range of $360^{\circ}$ at $12.5 \mathrm{GHz}$ is achieved with an maximum average phase error of $16^{\circ}$ for a phase dispersion of $60^{\circ} / \mathrm{GHz}$ in the band of interest 10.5-14.5 GHz.
\end{abstract}

\section{INTRODUCTION}

The synthesis of reflectarray consists in controlling individually the reflected phase on each unit cell to generate the desired phase law on the reflecting surface [1]. This is achieved by fixing the geometrical dimensions of each unit-cell. The current methods used to design reflectarrays are mostly based on full-wave analysis and so they are time consuming. An individual optimization of each unit-cell is hardly conceivable.

The objective of this work is to provide a quick sizing method for all cells in order to reduce the calculation time. The proposed solution consists in designing each unit-cell by using its associated equivalent circuit. This method has been used in designing frequency selective surfaces [2] microwave lenses [3] and recently reflectarrays antennas [4], [5].

The method proposed in this paper is based on [2] and [3] but is applied here to the design of single-layer reflectarray metallic-ring or slot-ring cells. In fact, it has been shown in [6] that such complementary cells allow to achieve a large phase range specification. Fig. 1 describes the method. It consists in converting the reflecting phase specification of a dipolar circuit (i.e. the equivalent circuit of a unit cell) into a transmission magnitude specification of a quadripolar circuit. The latter can then be easily synthesized with classical filtering techniques (e.g. Tchebycheff, ... ) [7] and the geometrical dimensions of the unit-cell deduced. The objective of this paper is to apply this method for configuring the respective equivalent dipolar circuit of metallic-ring and slot-ring cells.

\section{EQuivalent CiRcuit BASIS}

Fig. 2(a)(left) presents the structure of a single layer reflectarray unit-cell. The structure consists of a metallic layer printed on one side of a single dielectric substrate of thickness $h$ and a metallic ground plane on the other side. Its associated equivalent circuit is represented as a dipolar circuit in Fig. 2(a)(middle). The pattern is represented by a pure imaginary admittance $Y_{\text {Pattern }}$. The dielectric substrate is modeled by

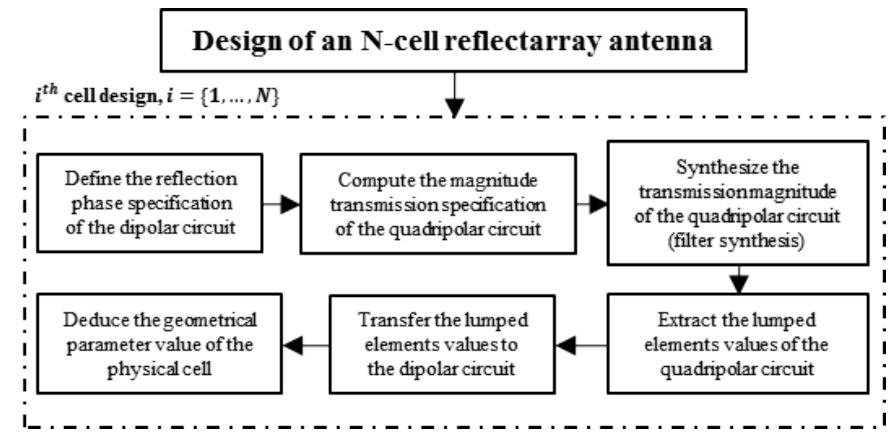

Fig. 1. General proposed synthesis procedure of a single layer reflectarray unit-cell.

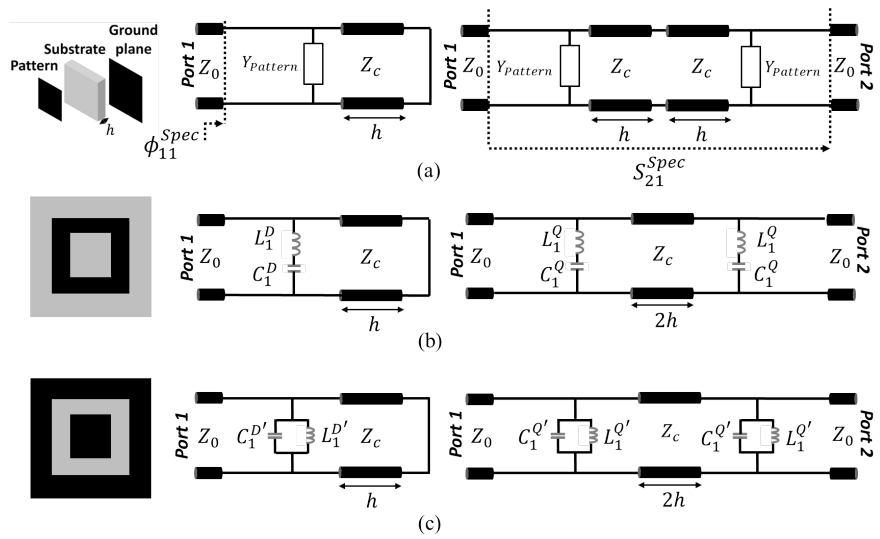

Fig. 2. Single-layer reflectarray unit cell, its dipolar circuit and its associated quadripolar circuit: general case (a), application to metallic-ring cell (b) and slot-ring cell (c).

a transmission line with length $h$ ended by a short circuit which models the metallic ground plane. The objective of the proposed method is to determine $Y_{\text {Pattern }}$ in order to respect the required reflection phase specification $\phi_{11}^{\text {Spec }}$ of the dipolar circuit. Fig. 2(a)(right) presents the symmetrical quadripolar circuit used to make the synthesis compatible with a classical filter synthesis. The reflection phase specification $\phi_{11}^{\text {Spec }}$ of the dipolar circuit is then converted into a magnitude transmission specification $S_{21}^{S p e c}$ of the quadripolar circuit through (1). 

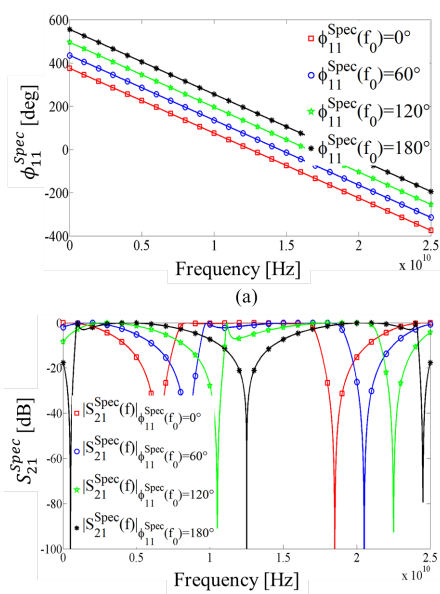

(c)

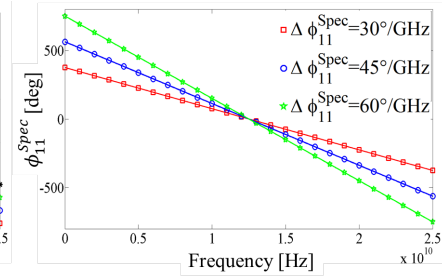

(b)

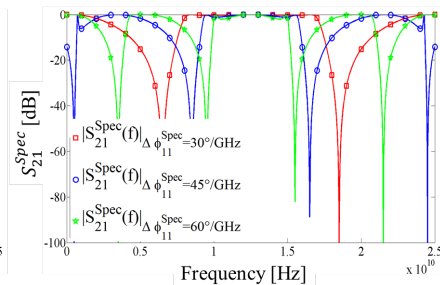

(d)
Fig. 3. Variation of the reflection phase specification $\phi_{11}^{S p e c}$ for different values of $\phi_{11}^{\text {Spec }}\left(f_{0}\right)$ (a) (for $\Delta \phi_{11}^{\text {Spec }}=30^{\circ} / G H z$ ), $\Delta \phi_{11}^{\text {Spec }}$ (b) (for $\left.\phi_{11}^{S p e c}\left(f_{0}\right)=0^{\circ}\right)$ and their respectively associated transmission magnitude specifications $\left|S_{21}^{S p e c}\right|_{d B}$ in (c) and (d).

$$
\begin{aligned}
& \left.\mid S_{21}^{\text {Spec }}(f)\right) \mid= \\
& \quad\left|\sin \left(\frac{2 \operatorname{atan}\left(\tan \left(\frac{\phi_{11}^{\text {Spec }}(f)}{2}\right)-\frac{2}{\sin (2 \beta h)}\right)-\phi_{11}^{S p e c}(f)}{2}\right)\right|
\end{aligned}
$$

Fig. 3 presents the reflection phase specifications, $\phi_{11}^{\text {Spec }}$, and their associated transmission magnitude ones $\left|S_{21}^{S p e c}\right|_{d B}$ in two cases and for a substrate thickness $h=\frac{\lambda_{0}}{4}$ at $f_{0}=12.5 \mathrm{GHz}$, the central frequency of our band of interest (10.5-14.5 GHz).

First, $\phi_{11}^{\text {Spec }}\left(f_{0}\right)$ varies from $-180^{\circ}$ to $180^{\circ}$ with a constant phase dispersion $\Delta \phi_{11}^{S p e c}=30^{\circ} / G H z$. Note that the magnitude specification behaves symmetrically according to the sign of $\phi_{11}^{\text {Spec }}\left(f_{0}\right)$. In the following, the results will then be presented for $\phi_{11}^{\text {Spec }}\left(f_{0}\right) \geq 0^{\circ}$ only (Fig. 3(a)). Fig. 3(c) shows that $\left|S_{21}^{S p e c}\right|_{d B}$ has then a third-order band-pass filter behavior with ripples in the passband and transmission zeros on each side of the passband when $\phi_{11}^{\text {Spec }}= \pm 180^{\circ}$. The passband shifts toward high frequencies as $\phi_{11}^{\text {Spec }}\left(f_{0}\right)$ increases (the shifting is toward low frequencies when $\phi_{11}^{\text {Spec }}\left(f_{0}\right)$ decreases). This shifting implies a magnitude dissymmetry on the ripple. In fact, if we focus only on the band of interest, the $\left|S_{21}^{S p e c}\right|_{d B}$ behavior goes from a symmetric band-pass filter $\left(\phi_{11}^{\text {Spec }}\left(f_{0}\right)=0^{\circ}\right)$ to a symmetric band-stop one $\left(\phi_{11}^{\text {Spec }}\left(f_{0}\right)=\right.$ $\left.180^{\circ}\right)$ through a set of non-symmetric band-pass or band-stop filters according to the transmission zeros position. Note that we observe the same behavior for $\phi_{11}^{\text {Spec }}\left(f_{0}\right) \leq 0^{\circ}$.

Second, $\phi_{11}^{\text {Spec }}\left(f_{0}\right)$ is now fixed $\left(0^{\circ}\right.$ in this example) and the phase dispersion $\Delta \phi_{11}^{\text {Spec }}$ varies from $30^{\circ} / G H z$ to $60^{\circ} / G H z$ (Fig. 3(b)). Fig. 3(d) shows that the bandwidth decreases when the phase dispersion increases. The bandwidth decreases as well in the case of a band-stop filter. Note that we observe the same behavior for $\phi_{11}^{\text {Spec }}\left(f_{0}\right) \leq 0^{\circ}$.

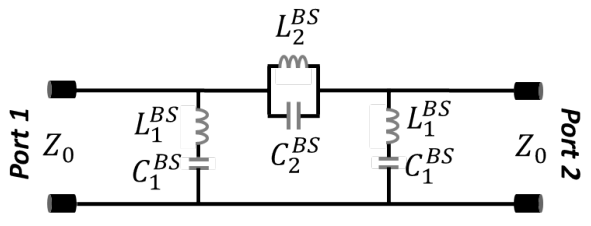

(a)

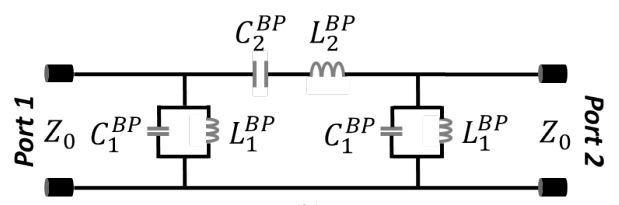

(b)

Fig. 4. Third-order band-stop (a) and band-pass (b) filter prototypes.

Finally, all responses can be approximated either by a thirdorder band-pass or band-stop Tchebycheff filter according to the band of interest. These prototype filters are used to configure the input and output resonators of the quadripolar circuits with a fixed $h$. Regarding the unit-cell, a band-stop behavior implies the use of a metallic-ring and a band-pass the use of a slot-ring. In the simplest cases, patches or square apertures can also be used. The two next sections present the synthesis procedure of their respective resonators.

\section{SYNTHESIS OF THE SERIES RESONATOR}

Fig. 2(b) depicts the top view of a metallic-ring unit-cell, its equivalent dipolar circuit and its associated quadripolar circuit. The metallic-ring is first modeled by a series resonator $\left(L_{1}^{D}\right.$, $C_{1}^{D}$ ) in parallel with a transmission line (length $h$, characteristic impedance $Z_{c}$ ) ended by a short-circuit. The dipolar circuit is then transformed into a symmetrical quadripolar circuit. Two identical series resonators $\left(L_{1}^{Q}, C_{1}^{Q}\right)$ are put in parallel upstream and downstream of a transmission line with length of $2 h$.

The magnitude behavior of the quadripolar circuit, $\left|S_{21}^{S p e c}\right|_{d B}$, is then approximated by a third-order band-stop filter (Fig. 4(a)) for a given reflection phase specification $\left(\phi_{11}^{\text {Spec }}\left(f_{0}\right), \Delta \phi_{11}^{\text {Spec }}\right)$. The band-stop filter prototype is synthesized to approximate the nearest transmission zero with respect to the band of interest. The first step to configure the filter prototype consists in deducing several parameters from the transmission magnitude specification $\left|S_{21}^{S p e c}\right|_{d B}$ as: the zero-transmission frequency $f_{0}^{S p e c}$; the cutoff frequency nearest to the passband $f_{1}^{\text {Spec }}$ (for $\phi_{11}^{\text {Spec }}\left(f_{0}\right)<0^{\circ}$ ) or $f_{2}^{\text {Spec }}$ (for $\phi_{11}^{\text {Spec }}\left(f_{0}\right)>0^{\circ}$ ) and the magnitude ripple $L_{a r}^{\text {Spec }}$ at the cutoff frequency. The second step consists in setting the bandstop prototype parameters (central frequency $f_{0}^{B S}$, ripple $L_{a r}^{B S}$, cutoff frequencies $f_{1}^{B S}$ and $f_{2}^{B S}$ ) as follows:

$$
\begin{gathered}
\left\{\begin{array}{c}
f_{0}^{B S}=f_{0}^{S p e c} \\
L_{a r}^{B S}=L_{a r}^{\text {Spec }}
\end{array}\right. \\
\left\{\begin{array}{l}
f_{1}^{B S}=f_{1}^{S p e c} \\
f_{2}^{B S}=\frac{f_{0}^{S p e c^{2}}}{f_{1}^{B S}} \text { for } \phi_{11}^{\text {Spec }}\left(f_{0}\right)<0^{\circ}
\end{array}\right.
\end{gathered}
$$


TABLE I

PHASE AND MAGNITUDE PARAMETERS FOR A $3^{r d}$-ORDER BAND-STOP FILTER APPROXIMATION

\begin{tabular}{|c|c|c|c|c|}
\hline \multirow{2}{*}{$\phi_{11}^{\text {Spec }}$} & Parameter & \multicolumn{2}{|c|}{$\phi_{11}^{\text {Spec }}\left(f_{0}\right)$} & $\Delta \phi_{11}^{\text {Spec }}$ \\
\cline { 2 - 5 } & Value & \multicolumn{2}{|c|}{$160^{\circ}$} & $30^{\circ} / G H z$ \\
\hline \multirow{2}{*}{$\left|S_{21}^{B S}\right|_{d B}$} & Parameter & $f_{0}^{B S}$ & $f_{2}^{B S}$ & $L_{a r}^{B S}$ \\
\cline { 2 - 5 } & Value & $11.8 \mathrm{GHz}$ & $12 \mathrm{GHz}$ & $-16.3 \mathrm{~dB}$ \\
\hline
\end{tabular}

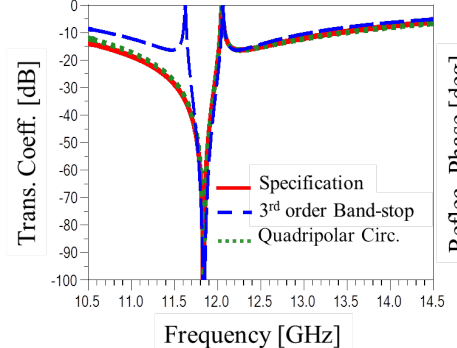

(a)

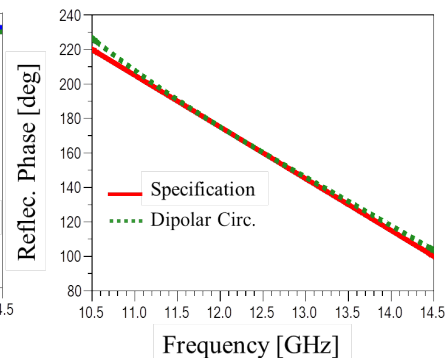

(b)
Fig. 5. Synthesis of a reflection phase specification $\phi_{11}^{\text {Spec }}$ presented in Table. I. Comparison between the transmission magnitudes (a) and reflection phases (b).

$$
\left\{\begin{array}{l}
f_{2}^{B S}=f_{2}^{S p e c} \\
f_{1}^{B S}=\frac{f_{0}^{S p e c}{ }^{2}}{f_{2}^{B S}} \text { for } \phi_{11}^{S p e c}\left(f_{0}\right)>0^{\circ}
\end{array}\right.
$$

The use of classical Tchebycheff band-stop synthesis [7] allows to determine $L_{1}^{B S}, C_{1}^{B S}, L_{2}^{B S}$ and $C_{2}^{B S}$. We then go back to the quadripolar circuit with:

$$
\left\{\begin{array}{l}
L_{1}^{Q}=L_{1}^{B S} \\
C_{1}^{Q}=C_{1}^{B S} .
\end{array}\right.
$$

An example of specifications in phase and magnitude is given in Table. I. Fig. 5(a) presents the response of the corresponding third-order band-stop filter, of the realized quadripolar circuit and of the initial specification in magnitude. The two latter match very well. Fig. 5(b) shows that the reflection phase of the realized dipolar circuit and the specification also match very well.

However, the band-stop filter approximation reveals some limitations for certain phase range specifications. Fig. 6(a) presents the results of the realized phase for several specifications where $\phi_{11}^{\text {Spec }}\left(f_{0}\right)$ varies from $0^{\circ}$ to $180^{\circ}$ with a constant phase dispersion $\Delta \phi_{11}^{\text {Spec }}=30^{\circ} / G H z$. As can be seen, the phase error between the realized phase and the specification one increases as $\phi_{11}^{\text {Spec }}\left(f_{0}\right)$ decreases from $180^{\circ}$ to $0^{\circ}$. This is actually due to the position of the approximated stop-band with respect to the band of interest. In fact, the transmission zero is shifted toward low frequencies and moved out from the band of interest as $\phi_{11}^{\text {Spec }}\left(f_{0}\right)$ decreases. The phase error depends also on the phase dispersion $\Delta \phi_{11}^{\text {Spec }}$. Fig. 7 shows the average phase errors (solid lines) between the realized phase and the specification one for three phase dispersions $\Delta \phi_{11}^{\text {Spec }}$ in the band of interest. It can be seen that, for a given $\phi_{11}^{\text {Spec }}\left(f_{0}\right)$, the average phase error increases as the phase dispersion $\Delta \phi_{11}^{\text {Spec }}$ increases. The average phase error remains under $16^{\circ}$ for a

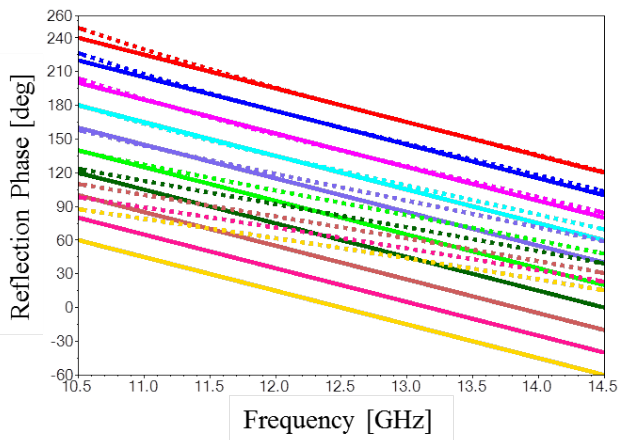

(a)

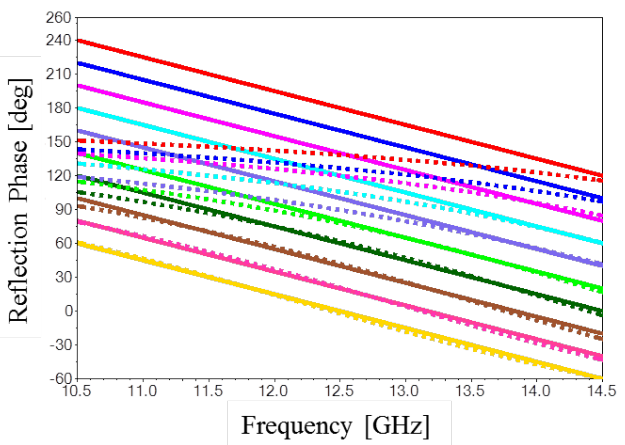

(b)

Fig. 6. Comparison between the phase responses of the realized dipolar circuit (dot lines) and the initial specification ones (solid lines) in the case of a thirdorder (a) band-stop filter approximation and (b) band-pass one .

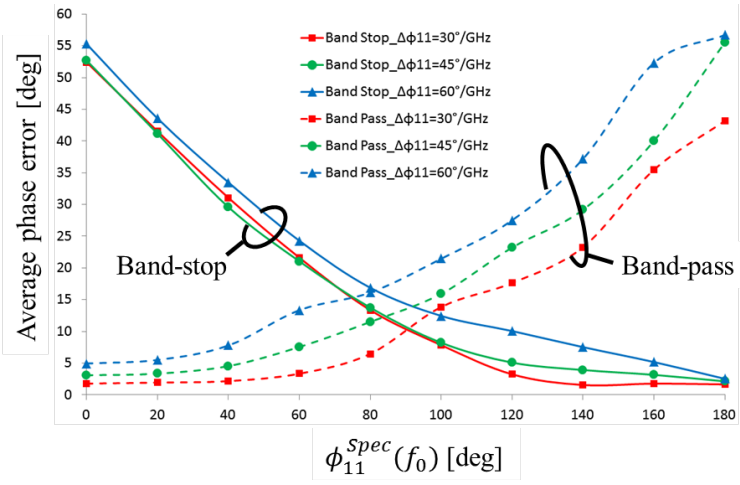

Fig. 7. Average phase error in the band of interest between the realized phase and the specification one for three dispersion phases $\Delta \phi_{11}^{S p e c}=$ $\left(30^{\circ} / \mathrm{GHz}, 45^{\circ} / \mathrm{GHz}, 60^{\circ} / \mathrm{GHz}\right)$ and in two cases: band-stop approximation and band-pass one.

range of $\phi_{11}^{\text {Spec }}\left(f_{0}\right)$ from $80^{\circ}$ to $180^{\circ}$ (and symmetrically from $-80^{\circ}$ to $-180^{\circ}$ ) for the three phase dispersions.

Eventually, the synthesis of a metallic-ring cell can be achieved by using a third-order band-stop filter synthesis only within this specific phase range. Nevertheless, in order to cover the complementary phase range, the dual cell (using a slotring) can be used as we will see now. 


\section{SYNTHESIS OF THE PARALLEL RESONATOR}

The slot-ring cell is studied in this section. Its topology, dipolar circuit and associated quadripolar circuit are depicted in Fig. 2(c). The slot-ring is modeled by a parallel resonator $\left(L_{1}^{D^{\prime}}, C_{1 S}^{D^{\prime}}\right)$ in the dipolar circuit and by two identical parallel resonators $\left(L_{1}^{Q^{\prime}}, C_{1}^{Q^{\prime}}\right)$ in its associated quadripolar circuit.

Here, the magnitude behavior of the quadripolar circuit, $\left|S_{21}^{S p e c}\right|_{d B}$, is approximated by a third-order band-pass filter (Fig. 4(b)) for a given reflection phase specification $\left(\phi_{11}^{\text {Spec }}\left(f_{0}\right), \Delta \phi_{11}^{\text {Spec }}\right)$. Unlike the band-stop filter approximation, the band-pass filter prototype is synthesized to approximate the pass-band of $\left|S_{21}^{S p e c}\right|_{d B}$. The first step here also consists in deducing several parameters from $\left|S_{21}^{S p e c}\right|_{d B}$ such as: the magnitude ripple, $L_{a r}^{S p e c}$, the minimum value in the case when $\phi_{11}^{\text {Spec }}\left(f_{0}\right) \neq 0^{\circ}$ and the cutoff frequencies $\left(f_{1}^{S p e c}, f_{2}^{S p e c}\right)$ measured at $L_{a r}^{S p e c}$. The central frequency is then calculated as follows:

$$
f_{0}^{S p e c}=\sqrt{f_{1}^{S p e c} f_{2}^{S p e c}} .
$$

The second step consists in setting the band-pass prototype parameters (central frequency $f_{0}^{B P}$, cutoff frequencies $f_{1}^{B P}$ and $f_{2}^{B P}$ and ripple $L_{a r}^{B P}$ ). These values are then respectively affected to the prototype parameters $\left(f_{0}^{B P}, f_{1}^{B P}, f_{2}^{B P}\right.$ and $\left.L_{a r}^{B P}\right)$. The use of classical Tchebycheff band-pass synthesis [7] allows to determine $L_{1}^{B P}$ and $C_{1}^{B P}$. We then go back to the quadripolar circuit and affected as well these values to $L_{1}^{Q^{\prime}}$ and $C_{1}^{Q^{\prime}}$. Note that a pre-distortion of the filter response (small shift in $f_{1}^{B P}$ and $f_{2}^{B P}$ ) is needed to better match the expected specifications.

An example of specifications in phase and magnitude is given in Table. II. Fig. 8(a) presents in this case the response of the corresponding third-order band-pass filter, of the realized quadripolar circuit and of the initial specification in magnitude. The two latter match very well. Fig. 8(b) shows that the reflection phase of the realized dipolar circuit and the specification also match very well.

However, as in Section. III, the band-pass filter reveals some limitations as well. Fig. 6(b) presents the results of the realized phase for several specifications (see. Section. III). As can be seen, the phase error increases as $\phi_{11}^{\text {Spec }}\left(f_{0}\right)$ increases from $0^{\circ}$ to $180^{\circ}$. This is actually due to the location of the pass-band with respect to the band of interest. In fact, it is shifted toward high frequencies and moved out from the band of interest as $\phi_{11}^{\text {Spec }}\left(f_{0}\right)$ increases. Fig. $7($ dot lines) shows the average phase error between the realized phase and the specification one for three phase dispersions $\Delta \phi_{11}^{S p e c}$ in the band of interest. It can be seen that, for a given $\phi_{11}^{\text {Spec }}\left(f_{0}\right)$, the average phase error increases as the phase dispersion $\Delta \phi_{11}^{S p e c}$ increases. The average phase error remains under under $16^{\circ}$ but for a range of $\phi_{11}^{\text {Spec }}\left(f_{0}\right)$ from $0^{\circ}$ to $80^{\circ}$ (and symmetrically from $0^{\circ}$ to $-80^{\circ}$ ) for the three phase dispersions.

\section{CONCLUSiON}

In this paper, a method for synthesizing single-layer reflectarray unit-cell based on filter synthesis techniques was
TABLE II

PHASE AND MAGNITUDE PARAMETERS FOR A $3^{r d}$-ORDER BAND-PASS FILTER APPROXIMATION

\begin{tabular}{|c|c|c|c|c|c|}
\hline \multirow{2}{*}{$\phi_{11}^{\text {Spec }}$} & Parameter & \multicolumn{2}{|c|}{$\phi_{11}^{\text {Spec }}\left(f_{0}\right)$} & \multicolumn{2}{|c|}{$\Delta \phi_{11}^{\text {Spec }}$} \\
\cline { 2 - 6 } & Value & \multicolumn{2}{|c|}{$20^{\circ}$} & \multicolumn{2}{|c|}{$45^{\circ} / G H z$} \\
\hline \multirow{2}{*}{$\left|S_{21}^{B P}\right|_{d B}$} & Parameter & $f_{1}^{B P}$ & $f_{2}^{B P}$ & $f_{0}^{B P}$ & $L_{a r}^{B P}$ \\
\cline { 2 - 6 } & Value & $8.6 \mathrm{GHz}$ & $19.8 \mathrm{GHz}$ & $13 \mathrm{GHz}$ & $-0.77 \mathrm{~dB}$ \\
\hline
\end{tabular}

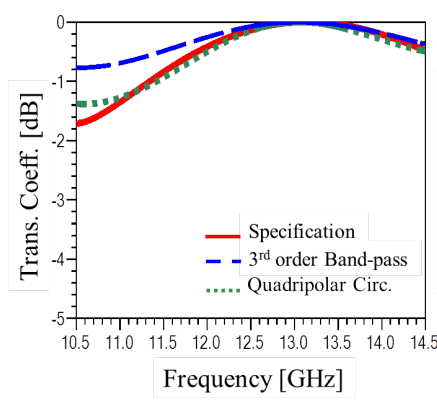

(a)

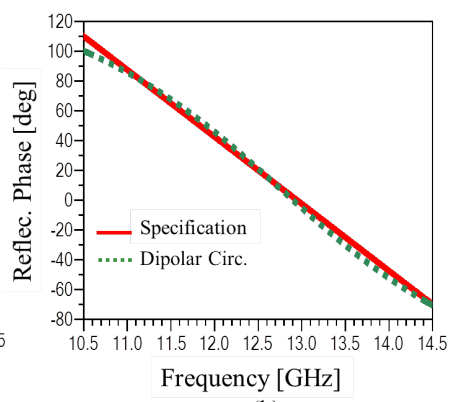

(b)
Fig. 8. Synthesis of a reflection phase specification $\phi_{11}^{\text {Spec }}$ presented in Table. II. Comparison between the transmission magnitudes (a) and reflection phases (b).

presented. The proposed method allows to convert a reflection phase specification on a dipolar circuit into a transmission magnitude one on a symmetrical quadripolar circuit. The latter can then be easily synthesized with classical filter synthesis techniques. Two complementary cells, a metallic-ring and a slot-ring, were studied in order to achieve a larger phase range specification. These cells were respectively synthesized using a $3^{r d}$-order band-stop and band-pass Tchebycheff filter. A phase range specification of $360^{\circ}$ at $12.5 \mathrm{GHz}$ was achieved with an average phase error, in the band of interest 10.5-14.5 $\mathrm{GHz}$, maintained under $16^{\circ}$ for a maximum phase dispersion specification of $60^{\circ} / \mathrm{GHz}$.

\section{REFERENCES}

[1] J. Huang and J. Encinar, Reflectarray Antennas. Wiley Inter-Sciences, 2008.

[2] M. A. Al-Joumayly and N. Behdad, "A generalized method for synthesizing low-profile, band-pass frequency selective surfaces with non-resonant constituting elements," IEEE Trans. Antennas Propag., vol. 58, no. 12, December 2010.

[3] _ "Wideband planar microwave lenses using sub-wavelength spatial phase shifters," IEEE Trans. Antennas Propag., vol. 59, no. 12, December 2011.

[4] A. Edalati and K. Sarabandi, "Reflectarray antenna based on grounded loop-wire miniaturised-element frequency selective surfaces," IET Microw. Antennas Propag, vol. 8, 2014.

[5] S. M. A. M. H. Abadi, K. Ghaemi, and N. Behdad, "Ultrawideband, true-time-delay reflectarray antennas using ground-planebacked, miniaturized-element frequency selective surfaces," IEEE Trans. Antennas Propag., vol. 63, no. 2, February 2015.

[6] T. Makdissy, R. Gillard, E. Fourn, E. Girard, and H. Legay, "A patch-slot combination approach for large band reflectarrays," EuRAD, November 2012.

[7] G. Matthaei, E. Jones, and L.Young, Microwave Filters, ImpedanceMatching Network, and Coupling Structures. Artech House, Inc., 1980. 